\title{
Communication Needs of Hispanic Patients: Sudden Speechlessness Simulation
}

\author{
Carmen S. Rodriguez ${ }^{1, *}$, Michelle S. Troche ${ }^{2}$ and Alexandra Johnson ${ }^{2}$ \\ ${ }^{1}$ College of Nursing, University of Florida, Box 100197 Gainesville, FL 32610, USA \\ ${ }^{2}$ Department of Speech, Language, \& Hearing Sciences, Division of Speech Pathology, University of Florida Box \\ 117420 Gainesville, FL 32611, USA
}

\begin{abstract}
The purpose of this study was to determine whether Spanish-dominant individuals had unique communication needs during a hypothetical event resulting in sudden speechlessness (SS), relative to an established template of messages with their respective graphic images/symbols previously validated by English-proficient patients experiencing sudden speechlessness. Spanish-dominant participants were included in a simulation where they became suddenly speechless while hospitalized after a surgical intervention. Participants listed basic care/symptom management needs important to communicate while speechless, identified corresponding illustrations by using a validated template, and/or described or drew pictorial representations appropriate to illustrate each of their needs. Study participants generated messages congruent with the experiences of English-dominant speechless participants by matching a list of messages with at least one picture or symbol included on the template. Participants generated new messages not represented in the original template to communicate about psychosocial needs, immediate needs, clarification/informational needs, and spiritual/faith needs. Results showed that the template in its original state did not meet the range of communication needs experienced by the Spanish-dominant study participants. Culturally specific input was essential to convey participants' communication needs during a period of speechlessness. Validation of study findings by Spanish-dominant individuals experiencing communication disorders will assist to further evaluate if messages and illustrations included on a revised template are representative of communication needs during SS periods.
\end{abstract}

Keywords: Communication needs, Sudden speechlessness, Hispanics.

\section{INTRODUCTION}

Sudden speechlessness, the sudden inability to verbalize needs, is associated with hospitalized patients recovering from head and neck surgery, trauma to structures critical to speech, or respiratory intubation. Communication breakdowns between suddenly speechless patients and health care providers can result in anxiety, fatigue, frustration, pain, and inefficient health care for patients, particularly when critical care needs emerge (Magnus \& Turkington, 2005). A language barrier, or limited English proficiency, adds complexity as speechless patients attempt to communicate their wants and needs in a nondominant language. Differences in most domains of communication have been identified between English-dominant and Spanish-dominant speakers. In particular, this has been found in the health care setting (Beach et al., 2010; Shoutent \& Meeuwesen, 2006; Weech-Maldonado, Fongwa, Gutierrez, \& Hays, 2008). Despite these communication problems and/or differences, there is little research on developing and validating useful tools for alternative/augmentative communication devices and strategies for Spanish-dominant patients. The need for a communication system that addresses these challenges of speechlessness has prompted this research to identify pictorial symbols and messages that are recognized as useful and sensitive by Spanish-dominant patients.

*Address correspondence to this author at the College of Nursing University of Florida Box 100197 Gainesville, FL 32610; Tel: 352-273-6328; Fax: 352-273-6536; E-mail: rodrics@ufl.edu
Issues of race, ethnicity, and culture have also been implicated as affecting quality of communication in the medical setting (Beach et al., 2010). Spanish-speaking patients typically feel that their physicians are less attentive and participatory in their care (2010). Barriers to communication between Spanish-speaking patients and Englishspeaking professionals can result in negative patient perceptions regarding timeliness of care, communication between patient and provider, and helpfulness of office staff (Weech-Maldonado et al., 2008). This may be attributed to lower expectations of service providers, racial biases, cultural misunderstandings, or cognitive-linguistic barriers (Shoutent \& Meeuwesen, 2006). Independent of the reasonning, research has noted that physicians treat Spanishspeaking patients with less perceived concern resulting in these patients being less verbally expressive (2006).

The ability to make appropriate health decisions is based on one's capacity to obtain, process, and understand basic health information (Nielson-Bohlman, Panzer, \& Kindig, 2004). This phenomenon, health literacy, has also been found to be adversely affected when primary language differences exist between patients and their health professionals (Sudore et al., 2009). Sudore et al. (2009) studied the health literacy of English speakers with Englishspeaking physicians (English-concordant), Spanish speakers with Spanish- speaking physicians (Spanish-concordant), and Spanish speakers with English-speaking physicians (Spanish-discordant). Sudore and colleagues aimed to 
compare receptive communication (comprehension of ideas), proactive communication (expression of concerns/ideas), and interactive communication (willingness to ask questions and converse with professionals), across language concordance and language type. The results revealed that poor health literacy directly correlated to poor patient comprehension and expression of health information (Sudore et al., 2009). Limited health literacy with Spanish-concordant and discordant patients was associated with poor interactive communication. This was not the case for English-concordant patients. Spanish speakers with Spanish-discordant physicians had the worst receptive, expressive, and interactive communication among all subgroups, regardless of their health literacy. This further supports the assumption that Spanish-speaking patients are less likely to interact, ask questions, and report needs when their dominant language is not shared by their health care providers.

Although limited, there is some preliminary evidence to suggest that speechless English- and Spanish-dominant communicators have varied needs relative to augmentative and alternative communication. Huer (2000) tested African American, Chinese, European American, and Mexican participants on interpretation of various pictorial images. Participants used a 7-point scale to measure how closely the images depicted the phrases describing them. Cross-cultural differences were found for all four groups within the three symbol sets tested. The results revealed that culture and life experience affect the way people perceive graphic symbols. This evidence for culturally specific augmentative and alternative communication (AAC) coincides with the research of Hetzroni and Harris (1996), which identified methods for creating and using culturally specific AAC. These researchers claimed that without culturally sensitive AAC devices, patients are denied access to culturally appropriate and relevant ways of behaving and thinking. AAC must include access to routines, rituals, and universally accepted language within the social and cultural structure of the patient's community.

Spanish-dominant, speechless patients require alternative communication methods that facilitate communication between them, medical staff, and caregivers. Of critical importance is the patient's ability to communicate difficulty breathing, immediate need for suctioning or blocked airway, pain, inadvertent disconnection of ventilators or oxygen, and/or bleeding from disconnected IV lines (Rodriguez, 2003). Research shows that speechless patients benefit from the use of AAC strategies incorporating the use of speech generating devices (Patak et al., 2006; Happ, Roesch, \& Kagan, 2005; Rodriguez \& Blischak, 2010; Rodriguez \& Rowe, 2010; Happ, Roesch, \& Garrett 2004; Miglietta, Bochicchio, \& Scalea, 2004; Costello, 2000). It is accepted that improving medical team communication methods reduces length of patient stay in the intensive care unit, improves patient satisfaction, and decreases levels of morbidity (Ahrens, Yancey, \& Kollef, 2003; Lilly, Sonna, Haley, \& Massaro, 2003). Currently, health care professsionals, including physicians, nurses, and speech-language pathologists, rely on interpreters or their own ingenuity to communicate effectively with Spanish-dominant speechless patients. However, techniques for communication between English-speaking health care providers and English- dominant speechless patients (i.e., mouthing words, writing tablets, and alphabet boards) are less effective and efficient when the patient is Spanish-dominant. The Hispanic population, which now exceeds 50 million people in the US according to the US Census Bureau (2010), is considered the largest and fastest growing minority group. Subsequently, Hispanics constitute a large percentage of patients being treated in US hospitals. It is thus imperative that options be explored to enhance communication between the medical team and Spanish-dominant speechless patients.

The goal of this pilot study was to determine whether Hispanics had unique communication needs relative to a previously established template of graphic images/symbols identified as useful for speechless English-speaking participants (Rodriguez, Rowe, Koeppel, \& Thomas, under review; Rodriguez \& Blischak, 2010). The aims of this pilot study were as follows: (1) identify messages which Hispanic individuals would want to communicate if suddenly speechless and in a hospital environment and (2) determine whether the previously tested template met the commu-nication needs of Hispanics. Participants (who were able to speak) were prompted by a simulated event in which they imagined themselves in a situation resulting in sudden speechlessness.

\section{METHODOLOGY}

An observational, descriptive study was conducted. The researcher's Institutional Review Board provided approval before study implementation. All study participants were voluntary, and they each signed a consent form. Individual interviews were conducted with Hispanic individuals (one interview per participant) to obtain data on the effectiveness of the previously established template. Potential participants were recruited through posted flyers with information about the study, and through face-to-face announcements at community agencies normally accessed by the Hispanic population.

\section{SAMPLE}

Participants representative of Hispanic countries/regions outlined in the US Census Bureau (2010) were recruited and selected, based on the following criteria: (1) 18 years of age or older, (2) ability to read and write English or Spanish, and (3) Hispanic origin. Individuals unable to follow instructions or actively participate in the study for at least 30 minutes were excluded. Those interested in participating were informed about the purpose of the study, their role in the study, associated time commitment, and potential risks and benefits of participating. A total of 52 participants completed the study.

Research staff proficient in both English and Spanish implemented the study. Based on participants' preference, $38 \%$ of the interview sessions were conducted in English, and $62 \%$ of the sessions were conducted in Spanish. Participants signed the consent form in English or Spanish based on a self-report about reading, writing, and understanding the English language and the participant's level of English proficiency (LEP). The definition of LEP from the Improving America's Schools Act of 1994 was used as guidance to determine LEP of study participants: 


\section{Hypothetical Suddenly Speechless Scenario}

You are waking up in the Intensive Care Unit after having throat cancer surgery. Because the cancer was very large, the surgeon had to remove your vocal cords, which are required for speech. A small opening was made in your neck, and a tube was introduced to help you breathe while you recover. You are not able to speak, eat, lie down flat, sit up, or move your head from side to side. The side rails of your bed are up at all times because you are not allowed to get out of bed.

Fig. (1). Vignette used to simulate a situation in which a patient finds himself or herself SS.

An individual: (A) who (i) was not born in the United State[s] or whose native language is a language other than English and comes from an environment where a language other than English is dominant... and who has sufficient difficulty speaking, reading, writing, or understanding the English language and who [se] difficulties may deny such individual the opportunity... to participate fully in our society (p 34 section [8]).

\section{INSTRUMENTS}

- Demographic Data Form: The research team used this instrument to collect baseline data, including age, gender, years of education, ethnicity, and history of speechlessness.

- Vignette: The investigator developed a hypothetical scenario (Fig. 1) and instructions for the research team to ensure consistent data collection.

Communication Needs During Hospital Stay: The investigator developed a tool to collect information generated by study participants about template images, symbols, or messages after introducing the vignette. Interrater reliability was established prior to study initiation to determine consistency among raters. The interrater reliability for the raters was found to be Kappa=.91-1.0. Each rater independently recorded ratings, and discrepancies were resolved through consensus.

\section{DATA COLLECTION}

After consent was obtained, research staff coordinated individualized interviews to facilitate data collection. First, demographic data were obtained. Following participants' completion of the demographic data form, the vignette (Fig. 1) was given in written form to each participant and read aloud by a member of the research staff. Afterwards, participants were allowed a brief period for questions related to the vignette, and the following steps were then implemented:

1. Research staff asked each participant to list (either written or orally) all the basic care/symptom management needs considered important to communi cate as a result of the theoretical situation presented in the vignette.
2. Each participant was then shown a paper template of messages with their respective graphic images or symbols validated by English-speaking patients with a history of SS (Rodriguez et al., in review; Rodriguez \& Blischak, 2010) to determine if each of the communication needs on his or her list (from Step 1) could be associated with a picture or symbol on the template. If no corresponding picture or symbol was identified, the participant was asked to describe or draw symbols/pictorial representations appropriate for the need. Additionally, research staff took notes about descriptions provided by participants.

3. Messages related to basic care, comfort, and urgent needs (validated by English- speaking SS patients) were read, and participants identified corresponding symbols or pictures on the paper template.

4. Each participant reviewed available symbols/pictures on the template to identify those found useful during a period of SS.

\section{DATA ANALYSIS}

Classification of data generated from this pilot study was based on categories derived from previous research evaluating communication needs of English-speaking SS patients, when appropriate (Rodriguez et al., under review; Rodriguez \& Blischak, 2010). These categories included the following: immediate needs, clarification/informational needs, psychosocial needs, other needs. Descriptive statistics were used to synthesize data regarding communication needs and pictorial representation identified by participants.

\section{RESULTS}

\section{Participant Characteristics}

The sample consisted of 52 participants. Fifteen Hispanic countries represented in the study were classified by the following regions: South America ( $n=27 ; 51.9 \%)$, Caribbean Islands $(n=13 ; 25.0 \%)$, and Central America $(n=11 ; 21.1 \%)$. One participant reported more than one country of origin $(n=1 ; 1.9 \%)$ and was not classified by region of origin. Participants were mostly female, with a mean age of 32 years (range, 19-73), and a mean for years of education equivalent to 15 years (range, 2-22). Most 
participants $(n=43,83 \%)$ reported no previous history of sudden speechlessness. Those with a previous history of speechlessness $(n=9,17 \%)$ reported limitations to verbalize needs for a brief period of time (range, 15 minutes to 7 days) as a result of the following: laryngitis/tonsillitis, oral surgery, removal of benign node, and short-term intubation.

Aim 1: To identify messages which Hispanic individuals would want to communicate if suddenly speechless and in a hospital environment.

\section{Communication Needs of Hispanics After Exposure to Hypothetical Suddenly Speechless Scenario}

Participants generated between 1 to 16 messages $\left(\mathrm{x}^{-}=\right.$ 5.9; $\mathrm{SD}=2.9$ ) considered important to communicate after exposure to the theoretical situation presented in the vignette. When possible, categories associated with the template of messages generated by English-speaking SS patients (Rodriguez et al., under review; Rodriguez \& Blischak, 2010) were used to classify messages generated by study participants (Fig. 2). A total of 288 messages were generated.

Participants associated a picture or symbol on the template with communication needs on their list. Participants' responses to the hypothetical scenario and request to develop a list of needs considered to be important to communicate during an SS period resulted in identification of multiple messages, which were incorporated on the initial template developed with feedback provided by English-speaking SS patients (Rodriguez \& Blischak, 2010). Ninety-seven percent of the messages incorporated on the template were included in the initial list generated by Hispanics after being exposed to the hypothetical scenario.

Hispanics most often selected messages associated with communicating immediate needs, basic care, clarification/informational needs, and psychosocial needs (Table 1). More than $50 \%$ of Hispanics identified the need for communication about pain after a hypothetical scenario was described, whereas only $27 \%$ of English-speaking patients actually recovering from SS identified pain as a communication priority. Similar discrepancies were identified for communicating toileting needs, clarifying health status (e.g., How am I doing? Can you tell me more about that?), and psychosocial needs (e.g., How I feel today, desire/ability to communicate with relatives).

\section{Correlation of Template Messages and Graphic Images/Symbols and Usefulness During a Period of SS}

Participants had the option of evaluating messages included on the template for potential use during a period of SS. After research staff read each one of the messages listed on the template, participants selected pictures and symbols from the template which were appropriate for each message. A range of $26.9 \%-100 \%$ of Hispanics considered messages read by the research staff important to add to their selection of messages ("not on original list prepared, but wanted") compared to a range of $72.7 \%-100 \%$ of English-speaking SS patients from previous study (Table $\mathbf{1}$ ).

A small percentage of the Hispanic study participants (Table 1) identified messages on the template they did not consider necessary (not on list/not wanted) for communicating needs. The participants' rationale for unnecessary messages included more than one message describing the same need or the pictorial representation for the message was not appropriate or needed improvement.

Aim 2. To determine whether the previously tested template with English-proficient patients met the communication needs of Hispanics.

\section{Identification of New Messages by Hispanic Study Participants}

Participants generated messages which were not represented on the original template, for the following categories: immediate needs, clarification/informational needs, psychosocial needs, and other needs (Table 2). The category spiritual/faith needs was also created to incorporate associated messages concerning spiritual needs.

Hispanic participants recommended the inclusion of messages to meet emotional, social, and spiritual needs not addressed by messages included on template. Approximately $25 \%$ of messages were associated with the need to communicate about distraction/entertainment (e.g., turning

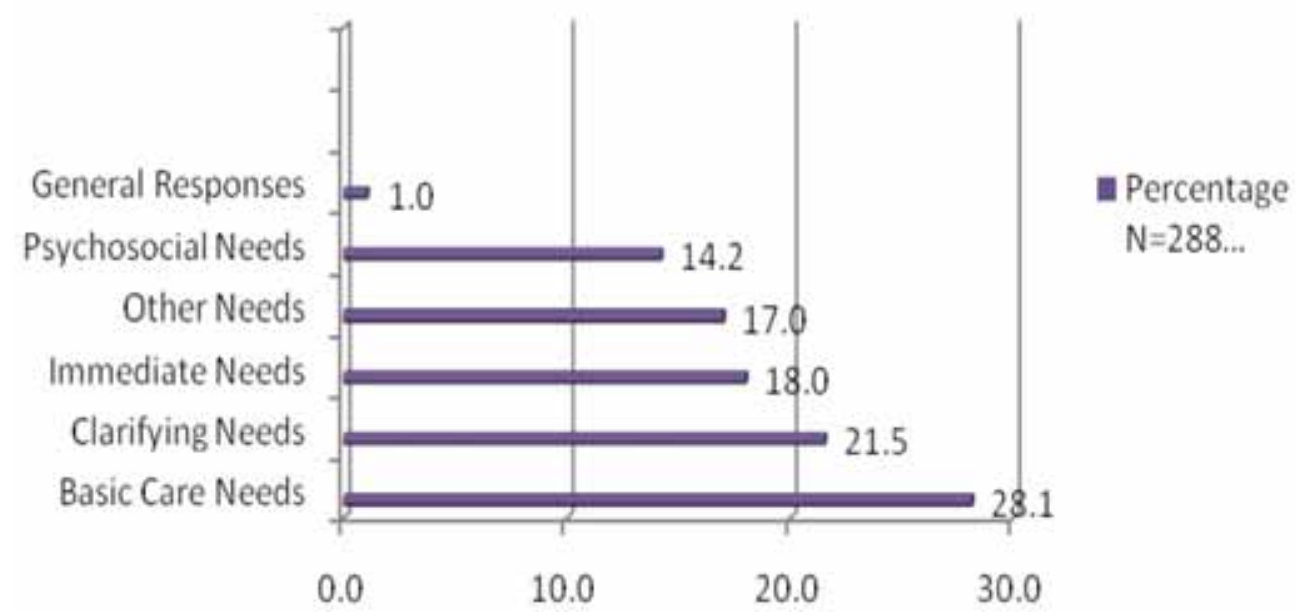

Fig. (2). Classification of messages generated by Hispanics after review of vignette. 
Table 1. Message Selection by Study Participants During a Period of Sudden Speechlessness

\begin{tabular}{|c|c|c|c|c|c|c|}
\hline \multirow[t]{2}{*}{ Messages on Template } & \multicolumn{3}{|c|}{ Hispanic Study Participants } & \multicolumn{3}{|c|}{ English-speaking SS Patients } \\
\hline & $\begin{array}{c}\text { List all Needs } \\
\text { Considered } \\
\text { Important to } \\
\text { Communicate } \\
\text { after SS Vignette } \\
\text { is Read. (Message } \\
\text { Generated by } \\
\text { Participant ) }\end{array}$ & $\begin{array}{l}\text { Message } \\
\text { Not on } \\
\text { List but } \\
\text { Wanted }\end{array}$ & $\begin{array}{l}\text { Message } \\
\text { Not on } \\
\text { List/ not } \\
\text { Wanted }\end{array}$ & $\begin{array}{c}\text { List all Needs } \\
\text { Considered } \\
\text { Important to } \\
\text { Communicate } \\
\text { During an SS } \\
\text { Period. } \\
\text { (Message } \\
\text { Generated by } \\
\text { SS Patient ) }\end{array}$ & $\begin{array}{l}\text { Message Not } \\
\text { on List but } \\
\text { Wanted }\end{array}$ & $\begin{array}{l}\text { Message } \\
\text { Not on } \\
\text { List/ Not } \\
\text { Wanted }\end{array}$ \\
\hline \multicolumn{7}{|l|}{ Immediate Needs } \\
\hline Stop & & $100 \%$ & - & $9.1 \%$ & $90.9 \%$ & - \\
\hline I have pain. & $55.8 \%$ & $44.2 \%$ & - & $27.3 \%$ & $72.7 \%$ & - \\
\hline I need a nurse now. & $25.0 \%$ & $75.0 \%$ & - & - & $100 \%$ & - \\
\hline I am nauseated. & $11.5 \%$ & $86.5 \%$ & $1.9 \%$ & - & $100 \%$ & - \\
\hline $\begin{array}{c}\text { I am having breathing } \\
\text { problems. }\end{array}$ & $9.6 \%$ & $86.5 \%$ & $3.8 \%$ & $18.2 \%$ & $81.8 \%$ & - \\
\hline I have trouble sleeping. & $7.7 \%$ & $88.5 \%$ & $3.8 \%$ & - & $81.8 \%$ & $18.2 \%$ \\
\hline I need suction. & $5.8 \%$ & $92.3 \%$ & $1.9 \%$ & $9.1 \%$ & $81.8 \%$ & $9.1 \%$ \\
\hline I want to rest now. & $5.8 \%$ & $94.2 \%$ & - & - & $100 \%$ & - \\
\hline Help & $3.8 \%$ & $94.2 \%$ & $1.9 \%$ & - & $81.8 \%$ & $18.2 \%$ \\
\hline Emergency & $3.8 \%$ & $90.4 \%$ & $5.8 \%$ & - & $90.9 \%$ & $9.1 \%$ \\
\hline \multicolumn{7}{|l|}{ General responses } \\
\hline Thank you. & & $100 \%$ & - & $9.1 \%$ & $72.7 \%$ & $18.2 \%$ \\
\hline Yes & 3.8 & $96.2 \%$ & - & $9.1 \%$ & $90.9 \%$ & - \\
\hline No & 3.8 & $96.2 \%$ & - & $9.1 \%$ & $90.9 \%$ & - \\
\hline \multicolumn{7}{|l|}{ Basic Care } \\
\hline $\begin{array}{l}\text { I need to go to the } \\
\text { bathroom. }\end{array}$ & $73.1 \%$ & $26.9 \%$ & - & $9.1 \%$ & $72.7 \%$ & $18.2 \%$ \\
\hline Drink & 38.5 & $59.6 \%$ & $1.9 \%$ & - & $100 \%$ & - \\
\hline I need the bedpan. & $32.7 \%$ & $59.6 \%$ & $7.7 \%$ & - & $100 \%$ & \\
\hline I need the urinal. & $26.9 \%$ & $65.4 \%$ & $7.7 \%$ & - & $81.8 \%$ & $18.2 \%$ \\
\hline Dry mouth & $7.7 \%$ & $88.5 \%$ & $3.8 \%$ & $14.3 \%$ & $85.7 \%$ & - \\
\hline Ice & $13.5 \%$ & $84.6 \%$ & $1.9 \%$ & $14.3 \%$ & $85.7 \%$ & - \\
\hline \multicolumn{7}{|l|}{ Clarification/Information } \\
\hline How am I doing? & $25.0 \%$ & $73.1 \%$ & $1.9 \%$ & $18.2 \%$ & $81.8 \%$ & - \\
\hline $\begin{array}{l}\text { Can you tell me more } \\
\text { about that? }\end{array}$ & $25.0 \%$ & $69.2 \%$ & $5.8 \%$ & $9.1 \%$ & $90.9 \%$ & - \\
\hline What is happening? & $15.4 \%$ & $80.8 \%$ & $3.8 \%$ & - & $100 \%$ & - \\
\hline I am doing OK. & $13.5 \%$ & $86.5 \%$ & - & - & $90.9 \%$ & $9.1 \%$ \\
\hline
\end{tabular}


Table 1. Contd....

\begin{tabular}{|c|c|c|c|c|c|c|}
\hline \multirow[t]{2}{*}{ Messages on Template } & \multicolumn{3}{|c|}{ Hispanic Study Participants } & \multicolumn{3}{|c|}{ English-speaking SS Patients } \\
\hline & $\begin{array}{c}\text { List all Needs } \\
\text { Considered } \\
\text { Important to } \\
\text { Communicate } \\
\text { after SS } \\
\text { Vignette is } \\
\text { Read. } \\
\text { (Message } \\
\text { Generated by } \\
\text { Participant ) }\end{array}$ & $\begin{array}{c}\text { Message } \\
\text { Not on List } \\
\text { but } \\
\text { Wanted }\end{array}$ & $\begin{array}{l}\text { Message } \\
\text { Not on } \\
\text { List/ not } \\
\text { Wanted }\end{array}$ & $\begin{array}{c}\text { List all Needs } \\
\text { Considered } \\
\text { Important to } \\
\text { Communicate } \\
\text { During an SS } \\
\text { Period. (Message } \\
\text { Generated by SS } \\
\text { Patient ) }\end{array}$ & $\begin{array}{l}\text { Message Not } \\
\text { on List but } \\
\text { Wanted }\end{array}$ & $\begin{array}{c}\text { Message } \\
\text { Not on } \\
\text { List/ Not } \\
\text { Wanted }\end{array}$ \\
\hline \multicolumn{7}{|l|}{ Psychosocial Needs } \\
\hline $\begin{array}{c}\text { Could you call my } \\
\text { relative? }\end{array}$ & $34.6 \%$ & $65.4 \%$ & - & $18.2 \%$ & $72.7 \%$ & $9.1 \%$ \\
\hline How I feel today & $19.2 \%$ & $78.8 \%$ & $1.9 \%$ & $9.1 \%$ & $81.8 \%$ & $9.1 \%$ \\
\hline I love you. & $7.7 \%$ & $90.4 \%$ & $1.9 \%$ & - & $90.9 \%$ & $9.1 \%$ \\
\hline \multicolumn{7}{|l|}{ Other needs } \\
\hline I am cold. & $15.4 \%$ & $84.6 \%$ & - & $9.1 \%$ & $90.9 \%$ & - \\
\hline I am hot. & $13.5 \%$ & $86.5 \%$ & - & - & $100 \%$ & - \\
\hline Pull me up in bed. & $19.2 \%$ & $71.2 \%$ & $9.6 \%$ & - & $83.3 \%$ & $16.6 \%$ \\
\hline
\end{tabular}

Note: Percentages reported are associated with number of participants selecting messages.

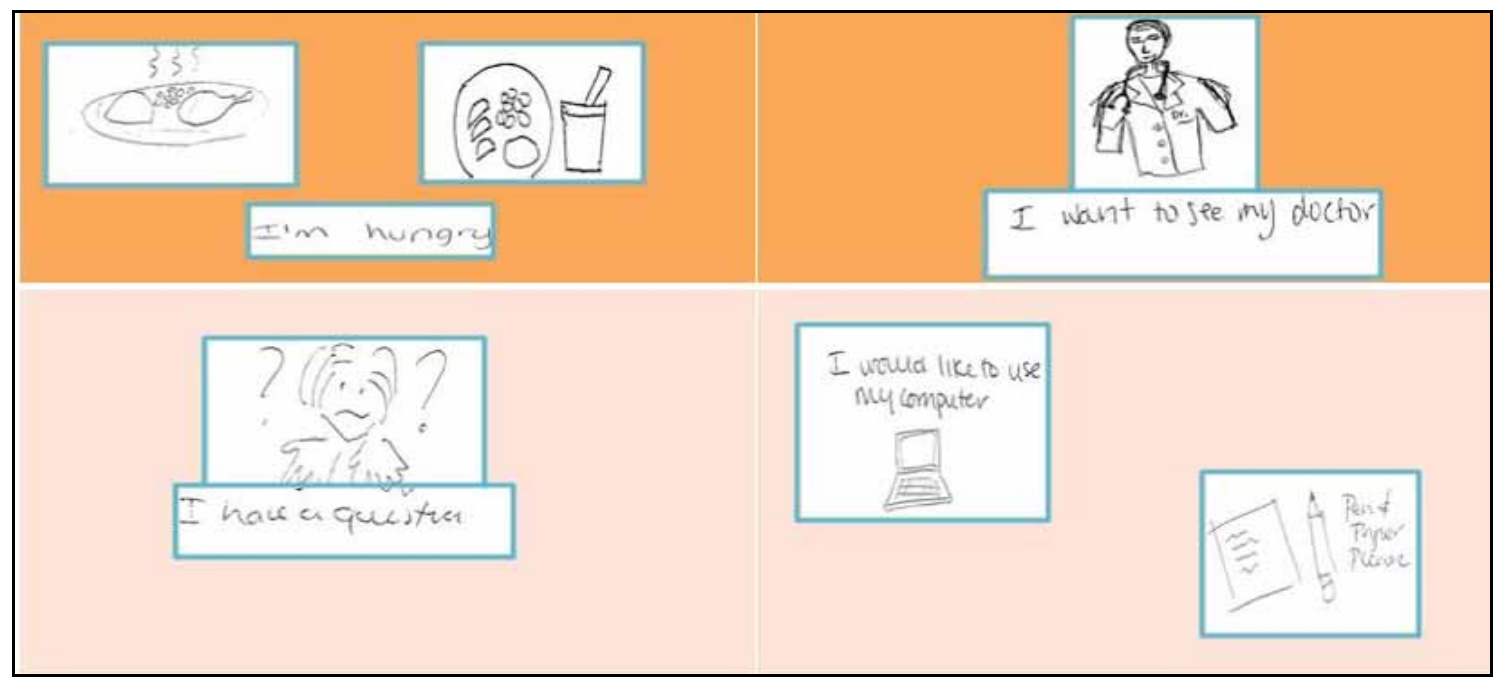

Fig. (3). Sample of new messages and illustrations recommended by Hispanic study participants to enhance template.

TV on, listening to music, reading a book). Other messages (range, 1.92\%-5.76\%) identified the need to communicate about emotions or feelings not addressed by English-speaking SS patients (e.g., boredom, desperation).

Hispanic study participants' recommendations were also made to communicate messages with a sense of immediacy such as symptoms ("Something itches"), need for nutritional intake ("I am hungry"), and/or communicating about the need to see a doctor ("I'd like to see the doctor now") (Fig. 3). Additionally, participants identified informational and spiritual needs that would potentially surface while speechless, and provided recommendations about core areas not addressed on the original template (Table 2).

\section{Pictorial Representation of Messages on Template Validated by English-Speaking SS Patients}

Participants $(n=52 ; 100 \%)$ were able to correlate at least one picture/symbol with messages read by research staff specific to basic care, comfort, and urgent needs. However, some participants $(n=13)$ considered it necessary to modify 
Table 2. Identification of New Messages by Hispanic Study Participants (n=52)

\begin{tabular}{|c|c|c|}
\hline Category & New Messages & Percentage $\%$ \\
\hline \multirow{8}{*}{ Psychosocial Needs } & Distraction/entertainment & $25.0 \%$ \\
\hline & Lonely & $5.76 \%$ \\
\hline & Desperate & $5.76 \%$ \\
\hline & Support/understanding & $3.85 \%$ \\
\hline & Boredom & $1.92 \%$ \\
\hline & Anxiety & $1.92 \%$ \\
\hline & Scared/confused & $1.92 \%$ \\
\hline & Anger & $1.92 \%$ \\
\hline \multirow{9}{*}{ Immediate Needs } & Thirst & $42.3 \%$ \\
\hline & Hunger & $42.3 \%$ \\
\hline & Need to see the doctor & $13.5 \%$ \\
\hline & Can't feel legs/leg cramps & $5.76 \%$ \\
\hline & Itching & $5.76 \%$ \\
\hline & Feeling extremely bad/not well & $3.85 \%$ \\
\hline & Dizziness & $3.85 \%$ \\
\hline & Tubes out of place & $1.92 \%$ \\
\hline & Vomiting & $1.92 \%$ \\
\hline \multirow{4}{*}{ Clarification/Informational Needs } & Health status/limitations after surgery & $42.3 \%$ \\
\hline & How to communicate while speechless & $15.4 \%$ \\
\hline & Treatment-related & $9.6 \%$ \\
\hline & Orientation to date, place or time & $7.7 \%$ \\
\hline \multirow{7}{*}{ Other Needs } & Increase in comfort & $17.3 \%$ \\
\hline & Hygiene needs & $7.7 \%$ \\
\hline & Environment control (light/noise) & $1.92 \%$ \\
\hline & Dry skin & $1.92 \%$ \\
\hline & Help with siderails & $1.92 \%$ \\
\hline & Noise & $1.92 \%$ \\
\hline & Greetings & $1.92 \%$ \\
\hline Spiritual/Faith & Spiritual support & $3.85 \%$ \\
\hline
\end{tabular}

pictures/symbols for various messages, while others recommended $(n=4)$ combining and/or removing a message, picture/symbol that appeared to convey similar needs.

Participants recommended certain modifications to drawings of symbols/images and related colors. They also recommended adding detail to some symbols/images to increase their clarity and adaptability for communicating needs. For instance, a modification to the icon representing family members included depicting extended family members such as the grandparent. Other modifications included (a) combining icons with the same theme (e.g., urinal, bedpan and toilet); (b) enhancing icons to better communicate a need for clarification and/or more information (e.g., emphasis of words depicted in icons"more information," "I have a question,"; (c) including arrows to depict position changes in bed; and (d) adding illustrations to depict emotional state (e.g., sad).

\section{DISCUSSION}

Communication differences between English and Spanish speakers have been well identified in the literature. In the medical setting, these differences can be detrimental to quality of care and health outcomes. Despite potential detriments, there have been few studies testing the utility of augmentative or alternative communication devices for Spanish-speaking populations. In the current study, a template of graphic images/symbols previously found to be useful for English-dominant speechless patients in the medical setting was tested in a group of Spanish-dominant participants. Study participants responded to a hypothetical scenario and successfully generated messages congruent 
with the experience of speechlessness as well as matched a list of messages with at least one picture or symbol included on the template. Results revealed that the template in its original state did not meet the range of communication needs experienced by Hispanic participants in this study.

Research suggests that including individuals from varying populations is important for identifying culturally relevant differences to improve health communication in target populations (Houts, Doak, Doak, \& Loscalzo, 2006; Huer, 2000). The communication needs identified by Hispanics required the use of messages and illustrations beyond those depicted on the original template tested in English-proficient SS patients. Although a majority of participants considered messages selected by Englishproficient SS patients appropriate for use during a period of SS, culturally specific input was essential for ascertaining whether messages and illustrations conveyed Hispanics' communication needs. For instance, the importance of integrating values shared among Hispanics was evident in recommendations to integrate diet, family structure, and spiritualism. Clearly, including Hispanics facilitated identification of culturally sensitive information to tailor and refine the existing template.

Most participants identified pain (56\%) as the most common immediate need to be communicated in response to the SS scenario. However, breathing issues, a problem commonly experienced by SS patients and a priority identified readily by English-proficient SS patients, were only identified in a small number of messages $(n=4 ; 8.8 \%)$ generated by Hispanics. This may be a limitation associated with this study, as Hispanic participants were responding to a hypothetical SS scenario rather than experiencing an SS event and associated complications. Validation of study findings with Hispanics experiencing SS and/or communication disorders that may result in SS should be considered in future studies.

In this study, participants' informational needs were associated with their health status, treatment, and management of the SS communication impairment. Even though participants were not actually recovering from an SS event, questions generated were appropriate, detailed, and emphasized the importance of communicating needs to others in the health care setting. In contrast, English-speaking SS patients identified informational needs as an important category but did not generate questions or provide details about their needs. It is unknown whether lack of proficiency in English influenced study results, thus it is possible that an increased number of questions may facilitate clarification and/or obtaining information during an SS event, especially when language proficiency limits ability to communicate effectively. Factors associated with this variation should be evaluated in future studies.

Language use variations among Hispanics have been reported, particularly regarding ways of speaking and configuring Spanish (Arrieta, 1994). In this pilot study, participants recognized the usefulness of the template, regardless of country of origin or differing versions of Spanish. Validation of this finding will be necessary with adequate representation of Hispanic countries underrepresented in this study.

\section{CONCLUSION}

Quality health care is synonymous with care that includes alternative or augmentative communication strategies to effectively address needs of a rapidly growing US population-Spanish-dominant Hispanics. Results from this pilot study provide information necessary for development of communication strategies both sensitive and useful for Hispanic patients who are rendered temporarily or permanently speechless as a result of surgery, trauma or or other events. The pilot data serve as an impetus for continued testing of culturally and linguistically appropriate communication interventions, which will benefit not only Hispanic patients but the physicians, nurses, speechlanguage pathologists, and other providers who care for them. Culturally sensitive tools that replace current ineffective/inefficient communication strategies (e.g., mouthing of words, writing tablets, and gestures) thus have great potential to significantly improve patient safety, efficiency of care, health care provider-patient relationships, and health outcomes for this population.

\section{ACKNOWLEDGMENTS}

This work was supported in part by NIH awards UL1RR029890, KL2RR029888, and a University of Florida College of Nursing Intramural Grant that were extended to Carmen S. Rodriguez. We thank Angelica Aguirre, Bryce Crouch, Liliana Mejia, and Maureen Connelly for their assistance.

\section{CONFLICT OF INTEREST}

Dr. Carmen Rodriguez, investigator on the study, is an inventor of communication software aimed at facilitating the communication process of hospitalized suddenly speechless patients.

\section{REFERENCES}

Ahrens, T., Yancey, V., \& Kollef, M. (2003). Improving family communications at the end of life: Implications for length of stay in the intensive care unit and resource use. American Journal of Critical Care, 12, 317-323.

Arrieta, O. (1994). Language and culture among Hispanics in the United States. In T. Weaver (Ed.), Handbook of Hispanic Cultures in the United States (pp. 168-190). Texas: Arte Publico Press.

Beach, M., Saha, S., Korthuis, P., Sharp, V., Cohn, J., Wilson, I., Eggly, S., Cooper, L., Roter, D., Sankar, A., \& Moore, R. (2010). Differences in patient-provider communication for Hispanic compared to nonHispanic white patients in HIV care. Journal of General Internal Medicine, 25, 682-687.

Costello, J.M. (2000). AAC intervention in the intensive care unit: The Children's Hospital Boston model. Augmentative and Alternative Communication, 16, 137-153.

Happ, M.B., Roesch, T.K., \& Garrett, K. (2004). Electronic voice-output communication aids for temporarily nonspeaking patients in a medical intensive care unit: A feasibility study. Heart Lung, 33, 92101.

Happ, M.B., Roesch, T.K., \& Kagan, S.H. (2005). Patient communication following head and neck cancer surgery: A pilot study using electronic speech-generating devices. Oncol Nursing Forum, 32, 1179-1187.

Hetzroni, O., \& Harris, O. (1996). Cultural aspects in the development of AAC users. Augmentative and Alternative Communication, 12, 5258 . 
Houts, P.S., Doak, C.C., Doak, L.G., \& Loscalzo, M.J. (2006). The role of pictures in improving health communication: A review of research on attention, comprehension, recall, and adherence. Patient Education and Counseling, 61, 173-90.

Huer, M. (2000). Examining perceptions of graphic symbols across cultures: Preliminary study of the impact of culture/ethnicity. Augmentative and Alternative Communication, 18, 267-273.

Lilly, C., Sonna, L., Haley, K., \& Massaro, A. (2003). Intensive communication: Four-year follow-up from a clinical practice study. Critical Care Medicine, 31, s394-s399.

Magnus, V., \& Turkington, L. (2005). Communication interaction in ICUPatient and staff experiences and perceptions. Intensive and Critical Care Nursing, 22, 167-180.

Miglietta, M.A., Bochicchio, G., \& Scalea, T.M. (2004). Computer-assisted communication for critically ill patients: A pilot study. The Journal of Trauma, 57, 488-493.

Nielson-Bohlman, L., Panzer, A., \& Kindig, D. (2004). Health Literacy: A prescription to end

confusion. Institute of Medicine. Washington D.C.: The National Academies Press.

Patak, L., Gawlinski, A., Fung, N.I, Doering, L., Berg, J., \& Henneman, E.A. (2006). Communication boards in critical care: Patients' views. Applied Nursing Research, 19, 182-190.
Rodriguez, C.S. (2003). Pain measurement in elderly head and neck cancer patients with communication impairments. Tampa, FL: University of South Florida.

Rodriguez, C.S., \& Blischak, D. (2010). Communication needs of nonspeaking hospitalized postoperative head and neck cancer patients. Applied Nursing Research, 23, 110-5.

Rodriguez, C.S., \& Rowe, M. (2010). Use of a speech-generating device for hospitalized postoperative patients with head and neck cancer experiencing speechlessness. Oncology Nursing Forum, 37,199205.

Rodriguez, C.S., Rowe, M., Koeppel, B. \& Thomas, L. (under review). Sudden speechlessness: Representing the needs of hospitalized patients. Schoutent, B., \& Meeuwesen, L. (2006). Cultural differences in medical communication: A review of the literature. Patient Education \& Counseling, 104, 21-34.

Sudore, R., Landefeld, C., Perez-Stable, E., Bibbins-Domingo, K., Williams, B., \& Schillinger, D. (2009). Unraveling the relationship between literacy, language proficiency, and patient-physician communication. Patient Educ Couns. 75, 398-402.

U.S. Census Bureau. (2010). State and County QuickFacts. Retrieved May 16, 2011, from http://quickfacts.census.gov/qfd/states/12000.html

Weech-Maldonado, R., Fongwa, M., Gutierrez, P., \& Hays, R. (2008). Language and regional differences in evaluations of Medicare managed care by Hispanics. Health Services Research, 43, 552568.

(C) Rodriguez et al.; Licensee Bentham Open.

This is an open access article licensed under the terms of the Creative Commons Attribution Non-Commercial License (http://creativecommons.org/licenses/by-nc/3.0/) which permits unrestricted, non-commercial use, distribution and reproduction in any medium, provided the work is properly cited. 